\title{
Thiourea Dioxide: A Multi-Purpose Reagent
}

\section{Roya Ayazi-Nasrabadi}

Bu-Ali Sina University, Hamedan, 6517838683, Iran r.ayazi.86@gmail.com

Published online: 09.04.2015

DOI: 10.1055/s-0034-1380498; Art ID: st-2015-v0513-v

\section{Introduction}

Thiourea dioxide (TUD), also known as formamidinesulfinic acid (FASA) or aminoiminomethanesulfinic acid (AIMS), is an organosulfur compound with white or pale yellow color; it is an odorless crystalline powder which is stable both in solid form and in cold aqueous solution. ${ }^{1}$ TUD is relatively soluble in water (at $20^{\circ} \mathrm{C}, 30 \mathrm{~g} / \mathrm{L}$ ) and insoluble in typical organic solvents and hence can easily be resumed and reused at the end of the reaction. ${ }^{2}$ Thiourea dioxide was first reported by Barnett in 1910. TUD can be prepared via the oxidation of thiourea by using hydrogen peroxide as an oxidant (Scheme 1). ${ }^{3}$
Roya Ayazi Nasrabadi was born in Kermanshah, Iran. She received her B.Sc. in chemistry (2007) and M.Sc. in organic chemistry (2010) from BuAli Sina University, Hamedan, Iran. Currently she works towards her Ph.D. under the supervision of Professor M. A. Zolfigol. Her research field is green chemistry, pharmaceutical compounds and catalysis, especially the application of homogeneous and heterogeneous catalysis in organic synthesis.

Recently, thiourea dioxide has been used in chemical and industrial processes, as multi-purpose reagent, ${ }^{4}$ catalyst, ${ }^{5}$ reductant. ${ }^{6}$ Thiourea dioxide is manufactured mainly for use in industrial processes such as discoloring dyes in paper recycling, textile printing and similar processes. ${ }^{7}$

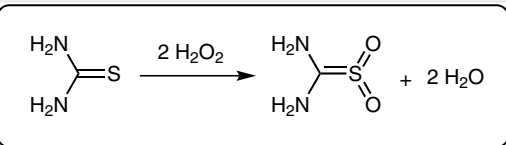

Scheme 1 Preparation of Thiourea Dioxide

Table 1 Use of Thiourea Dioxide: A Multi-Purpose Reagent

(A) PEG-embedded thiourea dioxide (PEG.TUD) as a novel organocatalyst:

Recently, Jain and co-workers reported two novel organocatalysts with urea and thiourea dioxide moieties. The host-guest complex between polyethylene glycol and thiourea dioxide (PEG.TUD) was prepared via both a co-crystallization method and a chemical reaction. The PEG.TUD complex is a very active and recyclable catalyst for the synthesis of 3,4-dihydropyrimidinones via the Biginelli method that supports high product yields. The corresponding poly(ethylene glycol)-thiourea complex, PEG.TU, is unreactive for the above-mentioned reaction. ${ }^{8}$

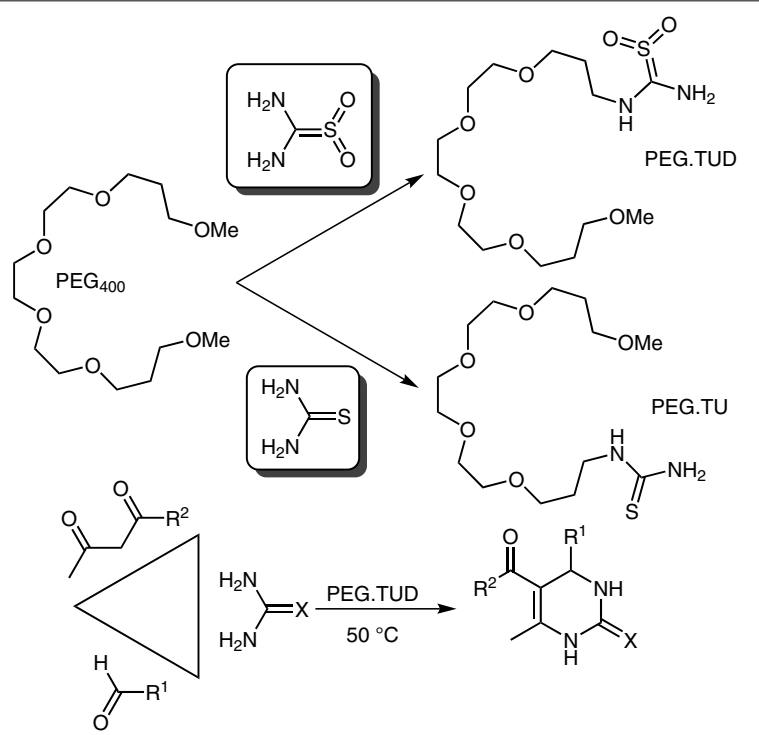


(B) Oxidation of sulfides to sulfoxides:

Kumar and co-workers introduced the first successful application of TUD as an efficient and highly selective organocatalyst for the oxidation of sulfides to sulfoxides with tert-butylhydroperoxide (TBHP) under mild reaction conditions. Thiourea dioxide has, owing to its simple availability, stability and strong hydrogen bonding capability, a great potential to activate oxygen donors through hydrogen bonding. ${ }^{9}$

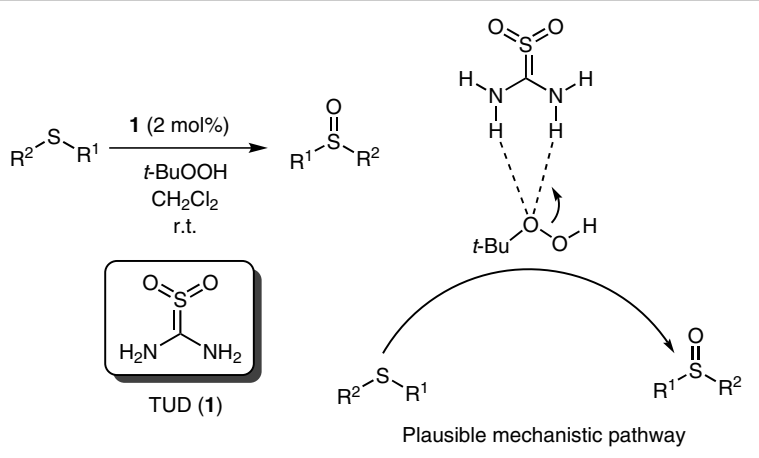

(C) Regio- and stereoselective ring-opening of epoxides:

A good range of $\beta$-hydroxy phenylselenides were synthesized via ring-opening reaction of mono-, di- and trisubstituted epoxides with the phenylselenide anion in the presence of a phase-transfer catalyst (PTC). Carrying out the reaction in aqueous $\mathrm{NaOH}-\mathrm{THF}$ using TUD led to higher yields. The simple generation of the products and the mild reaction conditions are remarkable aspects of the described method. ${ }^{10}$

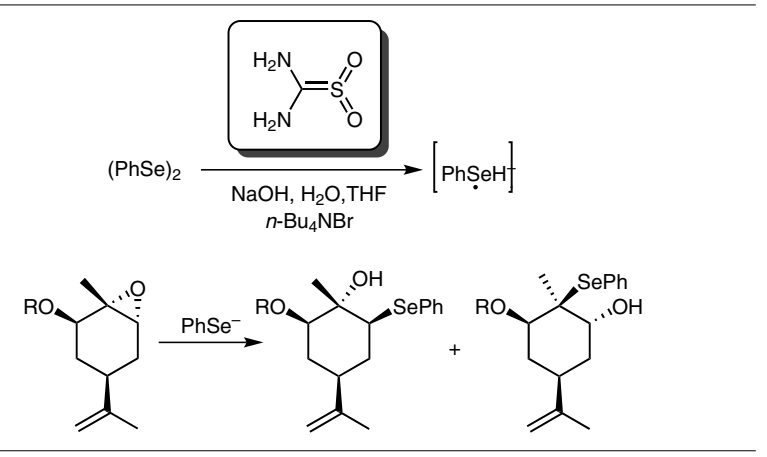

(D) Two-step one-pot synthesis of benzimidazoles:

A convenient method for the conversion of 2-nitroanilines into the corresponding benzimidazoles was reported. The method involves reduction of the nitro group, followed by intramolecular cyclization of the corresponding $o$-phenylenediamines utilizing thiourea dioxide and sodium hydroxide at $70{ }^{\circ} \mathrm{C}$ in $\mathrm{H}_{2} \mathrm{O}-\mathrm{EtOH}(\mathrm{v} / \mathrm{v}, 3: 1)$. The use of water as a green solvent, high yields and easy work-up are promising aspects of the reported protocol. ${ }^{11}$

\section{(E) Preparation of $\mathrm{N}$-Formamidinylamino Acids:}

Jursic et al. reported a practical and efficient synthetic procedure for the conversion of various amino acids into $\mathrm{N}$-formamidinylamino acids using formamidinesulfinic acids in basic water solution. It should be noted that the described method is suitable for the conversion of aliphatic amines into the corresponding $\mathrm{N}$-formamidinyl derivatives. ${ }^{12}$

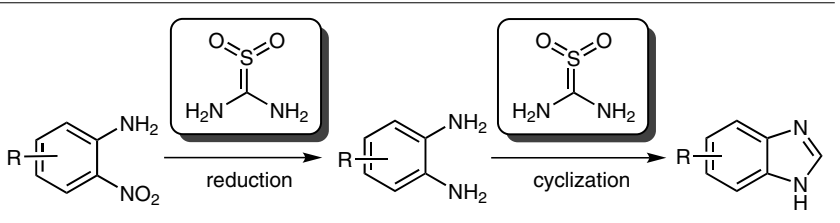

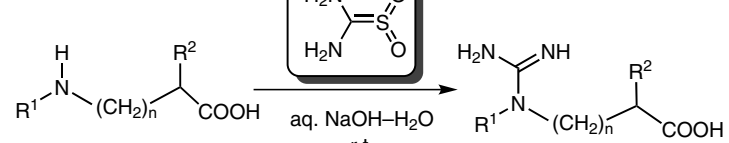

r.t.

\section{References}

(1) (a) Barnett, E. B. J. Chem. Soc., Trans. 1910, 97, 63. (b) Krug, P. J. Soc. Dyers Colour. 1953, 69, 606.

(2) Ghashang, M.; Mansoor, S. S.; Logaiya, K.; Aswin, K. Res. Chem. Intermediat. 2014, DOI: 10.1007/s-11164-014-1742-2.

(3) Ghashang, M.; Mansoor, S. S.; Aswin, K. Chin. J. Catal. 2014, 35 , 127.

(4) (a) Huang, W. Y.; Zhuang, J. L. Chin. J. Chem. 1991, 9, 270. (b) Kreutzberger, A.; Tesch, U. H. Arch. Pharm. 1978, 311, 429.

(5) (a) Wu, F. H.; Huang, B. N.; Lu, L.; Huang, W. Y. J. Fluorine Chem. 1996, 80, 91. (b) Verma, S.; Jain, S. L. Tetrahedron Lett. 2012, 53, 2595. (c) Verma, S.; Jain, S. L. Tetrahedron Lett. 2012, 53, 6055.
(6) (a) Abdel-Halim, E. S.; Al-Deyab, S. S. Carbohydr. Polym. 2011, 86, 1306. (b) dos Santos, R. B.; Brocksom, T. J.; Brocksom, U. Tetrahedron Lett. 1997, 38, 745. (c) Wang, J.; Zhou, T.; Deng, H.; Chen, F.; Wang, K.; Zhang, Q.; Fu, Q. Colloids Surf. B. 2013, 101, 171.

(7) Faebe, J.; Regitz, M. Römpp; Thieme: Stuttgart, 1990, 9th ed. 1426.

(8) Verma, S.; Jain, S. L.; Sain, B. Tetrahedron Lett. 2010, 51, 6897.

(9) Kumar, S.; Verma, S.; Jain, S. L.; Sain, B. Tetrahedron Lett. 2011, $52,3393$.

(10) dos Santos, R. B.; Juniora, V. L.; Zanottob, P. R.; Brocksom, T. J.; Brocksom, U. J. Chem. Res. 2008, 22.

(11) Pu, S.; Liang, Q.; Luo, X.; Luo, J. J. Chem. Res. 2014, 38, 118.

(12) Jursic, B. S.; Neumann, D.; McPherson, A. Synthesis 2000, 1656. 